\title{
The Effects of the Electroejaculation Procedure on Sperm Motility
}

\author{
T. Linsenmeyer, $M D,{ }^{1}$ C. Wilmot, $M D,{ }^{1}$ R. U. Anderson, MD, FACS ${ }^{2}$ \\ ${ }^{1}$ Department of Physcial Medicine and Rehabilitation, Santa Clara Valley Medi- \\ cal Center, San fose, California, ${ }^{2}$ Division of Urology, Stanford University \\ School of Medicine, Stanford, California, USA
}

\section{Summary}

Electroejaculation is becoming more available for obtaining semen from spinal cord injured men wishing to father children. This study was undertaken to determine what effect the electroejaculation procedure itself had on sperm motility. Factors which were found to impact on sperm motility were osmolarity, electric current and aqueous jelly lubricant used for bladder catheterisation. $p H$ did not affect sperm motility.

Key words: Infertility; Electroejaculation; Sperm motility.

There has been renewed enthusiasm for the use of electroejaculation to obtain semen from spinal cord injured (SCI) males. This arose because of a recent report of the first 6 pregnancies in the USA in which SCI men fathered children using this technique (Raymond, 1987).

This method of management for ejaculatory dysfunction is continuing to increase in popularity for several reasons. An increasing number of centres are performing electroejaculation, semen can be obtained from a majority of patients, and this is the safest method of obtaining an ejaculate in those prone to autonomic dysreflexia (Bennett et al., 1987; Brindley, 1981).

Because many SCI males have retrograde ejaculation, the electroejaculation procedure requires catheterising the bladder, draining it of urine, and instilling a more favourable physiologic $\mathrm{pH}$-buffered osmolar solution. This is an attempt to minimise the adverse effects of urine on sperm motility. Electrostimulation is then performed using a rectal probe. After an adequate current is delivered, the bladder is recatheterised and the buffered solution withdrawn and centrifuged to concentrate any sperm that might be present. Semen quality is then assessed. If the sperm are of adequate motility and number, they are used for artificial insemination.

Despite the ability to obtain an ejaculate, semen quality is often severely impaired following SCI. Sperm motility is most frequently affected. This has been attributed to the effects of urine on retrograde ejaculations, recurrent 
bacteriuria, stasis of prostatic fluid, scrotal hyperthermia, hormonal changes, and/or abnormal testicular histology (Perkash et al., 1985).

Because sperm motility often decreases following spinal cord injury, it is important to minimise any factors that could further inhibit motility. This study was undertaken to determine if any aspects of the electroejaculation procedure itself affect sperm motility.

\section{Methods}

This study specifically evaluated the effects on sperm motility of $\mathrm{pH}$, osmolarity, electric current, and aqueous lubricant used for catheterisation of the bladder.

Because of the poor sperm motility typically found in SCI males, and because SCI males' semen would have to go through the entire electroejaculation procedure in order to obtain an ejaculate, one or more ejaculates were obtained from 5 non-injured males. To determine the effects of $\mathrm{pH}$ and osmolarity, each semen sample was divided into aliquots. The aliquots were mixed with $2 \mathrm{cc}$ of saline. The osmolarity and $\mathrm{pH}$ were independently varied. The $\mathrm{pH}$ and osmolarity with the optimum motility for each patient's semen sample was then recorded. In this way each patient served as his own control. Specific solutions for this study were sterile saline and water for intravenous use at $152 \mathrm{mOsm}, 218$ mOsm, $253 \mathrm{mOsm}, 289 \mathrm{mOsm}, 444 \mathrm{mOsm}$, and $946 \mathrm{mOsm}$ at a constant $\mathrm{pH}$ of 7. Osmolarity was determined with a Dijimatic Osmometer model 302. $\mathrm{pH}$ was varied between 4 and 8 at a constant osmolarity of $289 \mathrm{mOsm}$. pH was adjusted prior to addition of semen aliquots by adding sodium bicarbonate or hydrochloric acid using a Corning $125 \mathrm{pH}$ meter.

Electric current was evaluated using a Neurometics Transcutaneous Nerve Stimulatory (TNS) unit, model number 660-01. Spermatozoa were suspended in a saline solution of $253 \mathrm{mOsm}$ at $\mathrm{pH} 7$. Motility was evaluated after 2 minutes of current at $100 \mathrm{~mA}$, pulse width $20 \mathrm{us}$, and pulse frequency of $20-30 \mathrm{~Hz}$ was applied. The result was then compared to controls.

Aqueous lubricant containing a bacteriostatic agent (1/15 000 parts of phenyl

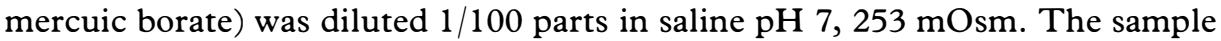
was suspended in this solution and sperm motility was determined and compared to samples suspended in saline without lubricant.

Spermatozoa motility for this study was evaluated using multiple exposure photography and a Neubauer haemocytometer. In the Figure the sperm tracts represent motile sperm and the bright spots are non-motile sperm. The motile and non-motile sperm were counted and per cent motility calculated for each sample.

\section{Results}

No obvious effect of $\mathrm{pH}$ on sperm motility was noted (Table I). Optimum motility was divided evenly for each $\mathrm{pH}$ of 4, 5, 6, 7 and 8. Osmolarity did appear to affect sperm motility (Table II). An osmolarity of $253 \mathrm{mOsm}$ produced the best motility the majority of the time. Current was also found to have an adverse effect on sperm motility (Table III). In every case sperm 


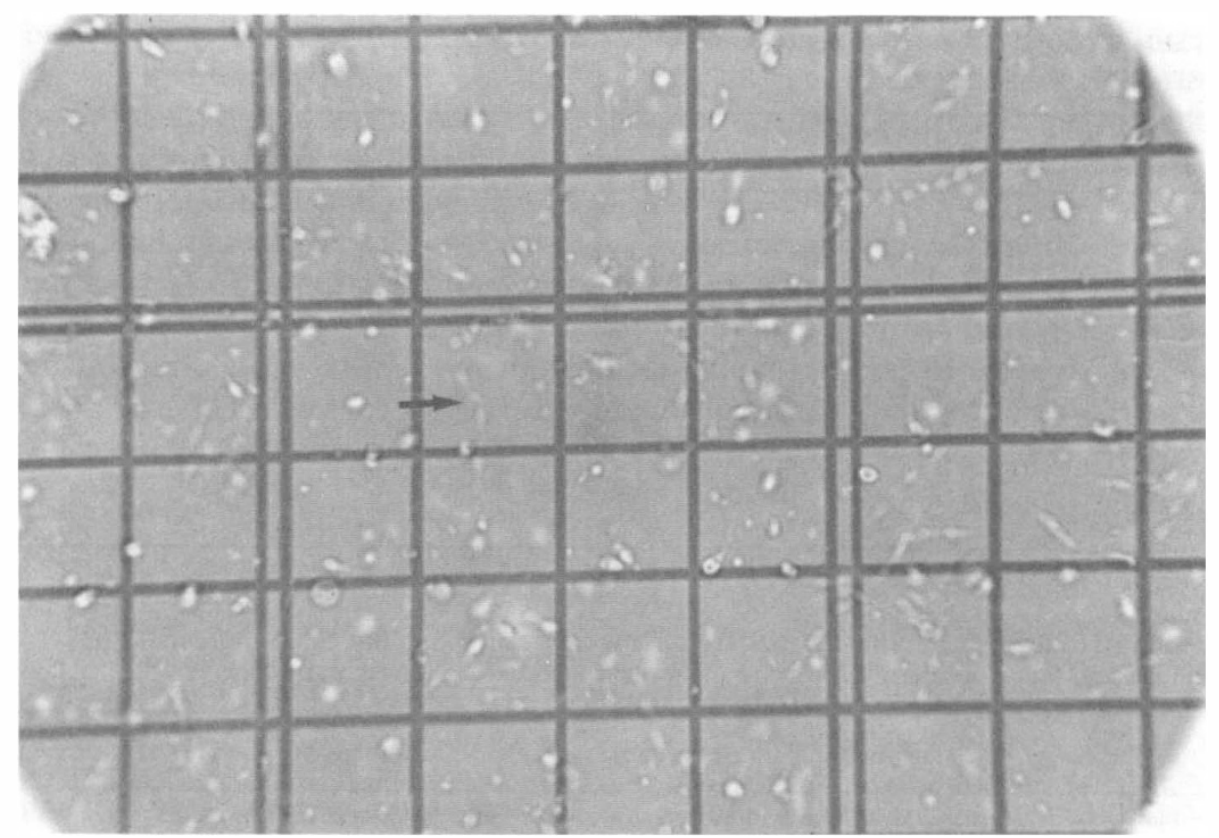

Figure Example of sperm motility evaluation with multiple exposure photography. The light tracks (arrow) are motile sperm. The bright dots are non-motile sperm.

Table I Effect of $\mathrm{pH}$ on sperm motility with constant osmolarity (284 mOSm). Results expressed as per cent motile sperm

\begin{tabular}{llllll}
\hline Sample & $\mathrm{pH}$ & 5 & 6 & 7 & 8 \\
\hline 1 & 4 & 37 & 41 & $46^{\star}$ & 44 \\
2 & 24 & 28 & $29^{\star}$ & 28 & 23 \\
3 & 24 & $15^{\star}$ & 14 & 12 & 2 \\
4 & 12 & 20 & 21 & 20 & $24^{\star}$ \\
5 & 23 & 20 & 21 & 19 & 20 \\
6 & $31^{\star}$ & 51 & 38 & 31 & 6 \\
7 & 5 & 25 & $53^{\star}$ & 52 & 52 \\
8 & 28 & 38 & 50 & $56^{\star}$ & 48 \\
\hline
\end{tabular}

${ }^{\star} \mathrm{pH}$ with best motility for each sample

subjected to a 2-minute current of $100 \mathrm{~mA}$, decreased motility when compared to controls. Within 5 minutes of exposure to the aqueous lubricant/saline solution $(1 / 100)$, sperm motilities dropped to $0-3^{\circ}{ }_{0}$ motility (Table IV).

\section{Discussion}

With improvements in both acute management and quality of life, a large number of SCI men have a strong desire to father children. This has become an important challenge for those working with the spinal cord injured. The use of electroejaculation is one way this challenge is being met. Care needs to be taken, however, that sperm is obtained in such a way that optimum motility is maintained. 
Table II Effect of osmolarity on sperm mobility with constant pH 7. Results expressed as per cent motile sperm

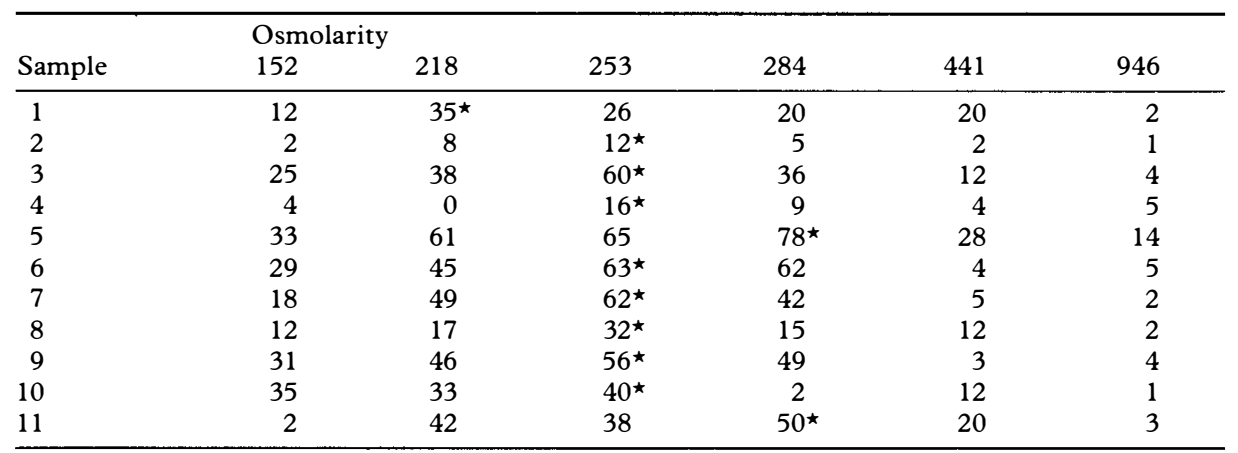

ऋ Osmolarity with best motility for each sample

Table III Effect of current (100 mA) for 2 minutes on sperm motility. Sperm suspended in saline at $\mathrm{pH} 7$ and osmolarity 284 mOsm. Results expressed as per cent motile sperm

\begin{tabular}{lcccccccc}
\hline & Sample & & & & & & \\
& 1 & 2 & 3 & 4 & 5 & 6 & 7 & 8 \\
\hline No current & 23 & 12 & 16 & 17 & 18 & 47 & 53 & 12 \\
100 mAmps & 2 & 10 & 1 & 14 & 14 & 34 & 22 & 6 \\
\hline
\end{tabular}

Table IV Effect of aqueous jelly lubricant on sperm motility. Sperm suspended in saline at $\mathrm{pH}$ 7 and osmolarity 253 mOsm. Aqueous jelly lubricant diluted 1:100 in saline

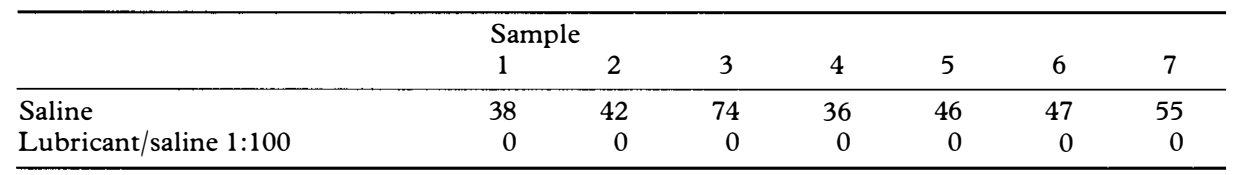

Urine is well known to have an extremely adverse effect on sperm. It has been noted that sperm motility may decrease by at least half within 5 minutes of mixing with urine (Crich et al., 1978). Traditionally this has been thought to be secondary to urine $\mathrm{pH}$, however, our results show that osmolarity should be the major concern when deciding on a solution to instill into the bladder prior to electroejaculation.

There is no published literature discussing the effects of electrical current on sperm motility. Preliminary results suggest that the current does adversely affect sperm motility. One problem in the field of electrojaculation is that there is no agreement on parameters for current delivered during electrostimulation. Horne, Paul and Munro, who did the initial work with electroejaculation in men, described an alternating current of 45 and $60 \mathrm{~mA}$ (1948). Brindley used a pulse generator with a peak electromotive force of $99 \mathrm{~V}, 100 \mathrm{msec}$ duration, 30 pulse/second frequency. Bennett et al. used an average of 30 stimulations of $15.6 \mathrm{~V}$ and $315 \mathrm{~mA}$ (1988). Further research on the effects of current on sperm motility may help to standardise these parameters.

Aqueous lubricant mixed with a $253 \mathrm{mOsm}$ sterile saline solution (1/100 parts) was found to have a significant adverse effect on sperm motility. It is important to note that these concentrations can easily be obtained within the 
bladder because of repeated catheterisations to drain the bladder of urine instilling small volumes $(20-30 \mathrm{cc})$ of buffer in the bladder during electroejaculation. Additionally, lubricant becomes concentrated in the centrifuged sperm pellet. We have found that saline solution alone provides adequate lubrication of the catheter for insertion in most cases.

Techniques of the electroejaculation procedure do appear to affect sperm motility. The most important effects were from osmolarity, current and aqueous lubricant used for catheterisation. Because SCI men often have poor semen quality, it is essential that the above factors be taken into consideration. They may make the difference in achieving pregnancy.

\section{References}

Bennett CJ, Seager SW, Vasher EA et al. 1987 Sexual dysfunction and electroejaculation in men with spinal cord injury: review. Fournal of Urology 139:453-457.

BRINDLEY GS 1981. Electroejaculation: its technique, neurological implications and uses. fournal of Neurological Neurosurgical Psychiatry 44:9.

CRICH JP, JEQUIER AM 1978 Infertility in men with retrograde ejaculation. Fertility and Sterility 30:572-576.

Horne HW Jr, Paul DP, MUnro D 1948 Fertility studies in the human male with traumatic injuries of the spinal cord and cauda equina. New England fournal of Medicine 239:959.

Perkash I, Martin DE, WeRner H 1985 Reproductive biology of paraplegics: results of semen collection, testicular biopsy and serum hormonal evaluation. Fournal of Urology 134:284-288.

RAYMOND CA 1987 New use of old method of inducing ejaculation may give hope of fatherhood to some spinal cord injured men. Fournal of American Medical Association 258:6:743-744. 